\title{
Consequences of Atrial or Ventricular Tachypacing on the Heat Shock Proteins (HSP) Level of Expression and Phosphorylation
}

\author{
Matthew Shorofsky*, Ange Maguy, Stanley Nattel
}

\begin{abstract}
Background - Uncontrolled atrial fibrillation (AF) results in complex changes in the cardiomyocyte electrical and contractile functioning that promote atrial remodeling and the continuation of AF. Recently there has been a growing interest in understanding the role of heat shock proteins (HSPs), which are cytoprotective molecular chaperones, in the pathophysiology of AF. Several groups have examined HSP expression in patients with AF but have yielded mixed results. To allow for better consistency and reproducibility between subjects, we utilized canine models to reproduce AFpromoting conditions to better investigate the role of HSPs in the pathophysiology of AF. Methods - AF promoting conditions were simulated in canine models with fifteen adult mongrel dogs $(20.6$ to $36.0 \mathrm{~kg})$ divided into three groups: (1) Control $(n=5)$, (2) two week ventricular tachypacing (VTP) induced congestive heart failure (CHF) $(n=5)$, and (3) one week atrial tachypacying (ATP) $(n=5)$. Quick frozen right atrial free wall tissue samples were used for protein isolation and were analyzed via Western blotting with data was expressed as a relative ratio and were analyzed using a two-tailed, unpaired ttest and significance was set at $\mathbf{p}<0.05$. The expression levels of HSP 90, 70, and 25 were studied along with the phosphorylation status of HSP27 at serine-78. Results - We first examined the effects of the ATP and CHF heart models on the expression of a select group of HSPs via Western Blot. We found that there was no significant difference in levels of expression of HSP 90, 70, or 25 when either ATP or CHF models were compared to control canines. The phosphorylation status of HSP27 was significantly decreased in the CHF canine model when compared to control $(p<0.0111)$ and it tended towards a decrease in the ATP canine model when compared to control $(\mathrm{p}=\mathbf{0 . 0 9 2 3})$. Conclusion - This study showed that even though the expression levels of HSPs may remain constant, there are protein phosphorylation and dephosphorylation events that occur in AF that may have important consequences in its pathophysiology. It is therefore necessary to investigate the full scale of HSP modifications during AF and AF-promoting conditions.
\end{abstract}

KEYWORDS: Atrial fibrillation, Heat shock proteins, Tachypacing, Phosphorylation

\section{INTRODUCTION}

Atrial fibrillation (AF) is the most common cardiac arrhythmia found in the general population and is associated with both morbidity and mortality (1). Poorly controlled AF can result in many complications such as stroke and tachycardia-induced cardiomyopathy, and according to the Framingham Heart Study, AF is associated with a decrease in overall survival (2). The maintenance of $\mathrm{AF}$ is promoted by AF-induced atrial

*To whom correspondence should be addressed:

Matthew Shorofsky

matthew.shorofsky@mail.mcgill.ca remodeling, which involves complex changes in the cardiomyocyte electrical and contractile function (3). The proteins involved in AF-induced atrial remodeling, and their respective molecular mechanisms are currently being delineated. One group of proteins, known as the heat shock proteins (HSPs), are molecular chaperons that are induced by many physiological stressors, and are known to convey a protective function in cells during times of stress (4). The HSPs are proteins of interest that play a key cytoprotective role in AF and may represent a novel molecular target in AF therapy. However, the underlying mechanism of their action in AF pathogenesis remains unclear. 
There has been growing interest in understanding the role of HSPs in the pathophysiology of AF. Recently, several groups have examined HSP expression in patients with AF, relative to those in sinus rhythm (SR), but these studies yielded mixed results. Although HSP expression was often found to be increased in patients with AF, there has been no clear consensus as to which groups of HSPs (HSP90, HSP70, HSP60, HSP27) are most effected (5-7). A more recent study indicated that ischemia-induced AF is prevented by the induction of exogenous HSP, and suggested that atrial ischemia in itself may activate HSP production (8).

Patients with AF experience a wide variation of severity and duration of symptoms. This degree of variability between subjects makes previous studies with only a limited number of human subjects unreliable and therefore it offers a possible explanation for why there has been no agreement in which HSPs are involved in the pathophysiology of AF. To allow for a better understanding of the pathophysiology of AF, it is therefore necessary to utilize an animal model which has the added benefit by allowing for control over the AF promoting conditions. In this study, AF-promoting conditions were simulated in canine models by two week ventricular tachypacing (VTP), which is a canine model of congestive heart failure (CHF), and by atrial tachypacing (ATP). Both atrial and ventricular tachypacying in canines is known to produce conditions conducive to atrial remodeling similar to that found in AF. In this current study the expression of HSP70, HSP90, and HSP25 were analyzed in right atrial tissue samples. Given that the phosphorylation status of small HSPs, such as HSP27 and HSP25 (HSP25 is an ortholog of HSP27), is crucial in the regulation of their function and cellular localization, the phosphorylation status of HSP27 was evaluated in both AF-promoting canine models as a means to further elucidate the role of HSPs in AF cytoprotection in vivo.

\section{METHODS}

\section{Animal Model and Cardiomyocyte Isolation}

The animal model was prepared as previously described (9). In brief, fifteen adult mongrel dogs (20.6 to $36.0 \mathrm{~kg}$ ) were divided into three groups: (1) Control $(n=5),(2)$ two week ventricular tachypacing (VTP) induced congestive heart failure $(\mathrm{CHF})(\mathrm{n}=5)$, and $(3)$ one week atrial tachypacying (ATP) $(n=5)$. CHF dogs had unipolar pacing leads inserted fluoroscopically into the right ventricular apex, which were programmed at $240 \mathrm{bpm}$ for 2 weeks. ATP remodeling was induced by 1 week of right atrial pacing at $400 \mathrm{bpm}$ after AV node ablation as previously described (10). The experimental protocols were reviewed and approved by the Montreal
Heart Institute Animal Research Ethics Committee.

On the final day of study the dogs were anesthetized with morphine (2 mg/kg SC) and alpha-chloralose (120 $\mathrm{mg} / \mathrm{kg} \mathrm{IV}$, followed by $29.25 \mathrm{mg} / \mathrm{kg}$ per hour) and mechanically ventilated. Right and left atria preparations were dissected and fast-frozen (9). RA and LA cells were stored separately in Tyrode solution with $200 \mu \mathrm{mol} / \mathrm{L} \mathrm{Ca} 2+$. In this study, right atrial samples were used.

\section{Protein Isolation}

Quick frozen right atrial free wall tissue samples were used for protein isolation. Tissue samples were placed in Ripa buffer (1\% IGEPAL, $0.5 \%$ Sodium deoxycholate, $0.1 \%$ SDS, and Beta-mercaptoethanol) containing protease inhibitors (Complete mini, EDTA free protease inhibitor cocktail tablets from ROCHE) and phosphatase inhibitors (sodium fluoride, betaglycerol phosphate, sodium orthovanadate, microcystin, EGTA, and EDTA). Samples were homogenized and placed on ice for 20 minutes and centrifuged at 3000 rpm for $10 \mathrm{mins}$ at $4^{\circ} \mathrm{C}$. The resultant supernatant was collected and centrifuged at $15000 \mathrm{rpm}$ for 15 minutes at $4^{\circ} \mathrm{C}$. The final supernatant was collected and lysate concentrations were quantified using the Bradford Assay.

\section{Western Blot Analysis}

Tissue extracts $(30 \mu \mathrm{g})$ were separated on a $10 \%$ SDS-polyacrylamide gel. Proteins were transferred to a polyvinylidene difluoride membrane (Millipore, Bedford, MA) and blocked for 1 hour at room temperature using 5\% milk in TBST. Membranes were incubated with primary antibody (1:2000; anti- HSP25, HSP70, HSP90, P-ser78 HSP27 from StressGen) over night at $4{ }^{\circ} \mathrm{C}$. The blots were then washed 3 times for 15 minutes with TTBS and then incubated for $1 \mathrm{~h}$ with the appropriate HRP-conjugated antibody (1:10000; antirabbit and anti-mouse). The blots were washed four times with TTBS. Immuno-complexes were detected using ECL and visualized on film. Bands were quantified via densitometry and standardized to GAPDH.

\section{Statistical Analysis}

Data are expressed as a relative ratio and were analyzed using a two-tailed, unpaired t-test and significance was set at $\mathrm{p}<0.05$.

\section{RESULTS}

\section{Relationship between ATP and CHF Canine Models and HSP Expression}

Canine models were utilized to provide a greater degree of standardization and reproducibility not possible when studying tissue samples from patients. We first examined the effects of the ATP and CHF heart 
A)
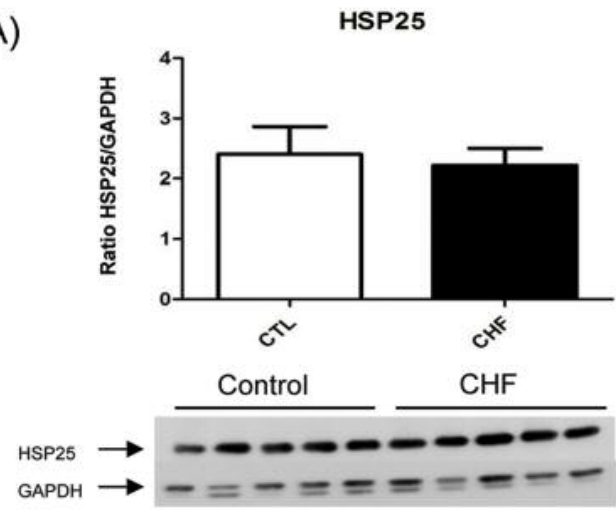

HSP25

B)

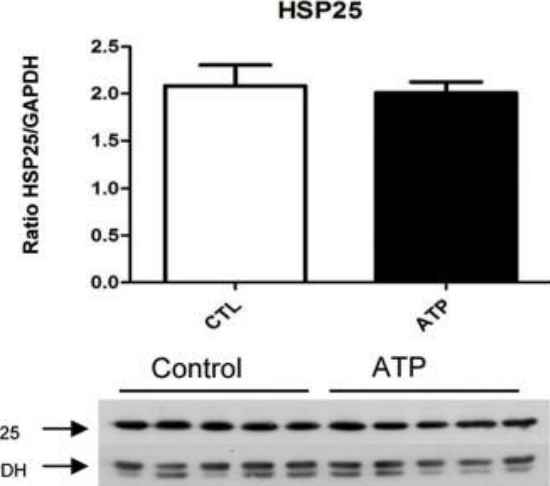

Figure 1: Graphs show the relative expression of HSP25, along with the corresponding Western Blots in control dogs $(n=5)$ versus A) Ventricular tachypacing model of CHF $(n=5), p=0.7405$ and B) ATP $(n=5), p=0.7728$. Protein expression levels were unchanged in either ATP or CHF models.

models on the expression of a select group of HSPs via Western Blot. With an $n=5$ for each of the control, ATP and $\mathrm{CHF}$ groups we found there was no significant change in expression levels of HSP25 in Control vs. CHF and Control vs. ATP (Figure 1). We also examined expression levels of other HSPs which have previously been shown to be related with AF. Analysis of expression levels of HSP70 (Figure 2) and HSP90 (Figure 3) again showed no significant difference between the experimental dogs (ATP and CHF) and the control dogs.

\section{HSP27 Phosphorylation Status is Reduced by ATP and $C H F$}

HSP27 has three known phosphorylation sites (ser15, ser78, and ser82) (11). The phosphorylation status of HSP27 is known have effects on cellular localization along with regulating its function as a molecular chaperone. To determine whether the phosphorylation status of HSP27 is affected in ATP and/or CHF canine models we examined the phosphorylation state on serine-78, which is known to be an important phosphorylation site in determining HSP27's cellular location (11). Phosphorylation was analyzed via
Western blot with control, ATP, and CHF on the same 15 well gel with $\mathrm{n}=5$ for each group. As seen in Figure 4, there was a trend towards decreased HSP27 phosphorylation in ATP, relative to the controls, although it failed to reach significance $(\mathrm{p}=0.0923)$. However, The CHF group, however, showed a significant decrease in the phosphorylation of HSP27 compared to the controls $(\mathrm{p}<0.0111)$.

\section{DISCUSSION}

To date the effects of AF-promoting conditions on HSP27 phosphorylation status has not been widely studied. We demonstrated in this study that the phosphorylation status of HSP27 is decreased in both the CHF and ATP canine models of AF. It is known that the phosphorylation of two serine residues (ser-78 and ser-82) is necessary for HSP27 localization into the nucleus (11). During conditions of stress, HSP27 is phosphorylated and translocated into the nucleus where it may protect against DNA damage (11). Also, dissociation of HSP27 oligermers by phosphorylation has been shown to stabilization F-actin and inhibit of actin degradation (12). This evidence suggests that phosphorylation is needed for HSP27 to convey a protective function. Our study showed that

A)
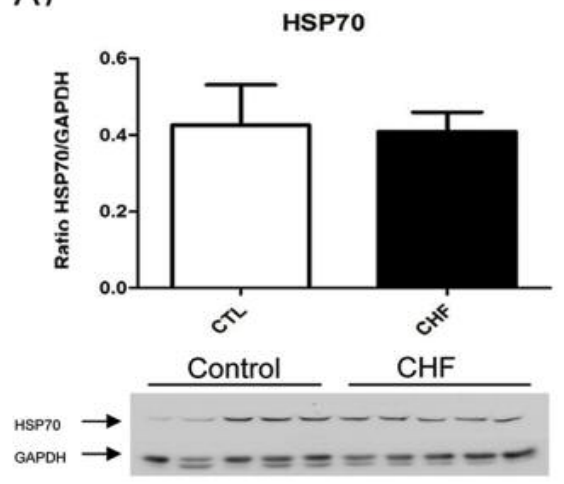

B)
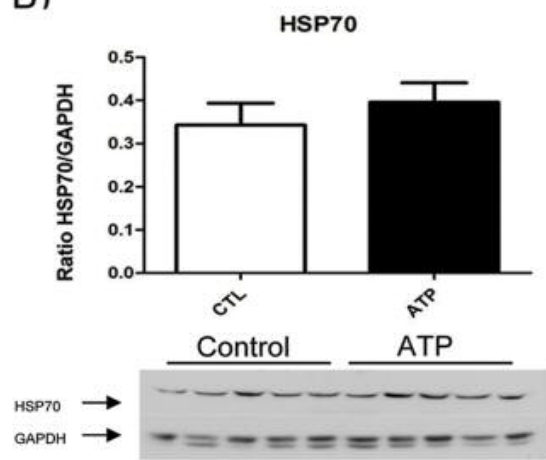

Figure 2: Western Blots and corresponding data showing the relative expression of HSP70 in control dogs ( $\mathrm{n}=5)$ versus A) Ventricular tachypacing model of CHF $(n=5), p=0.8874$ and B) ATP $(n=5)$, $\mathrm{p}=0.4597$. Protein expression level were unchanged in either ATP of CHF models. 
A)
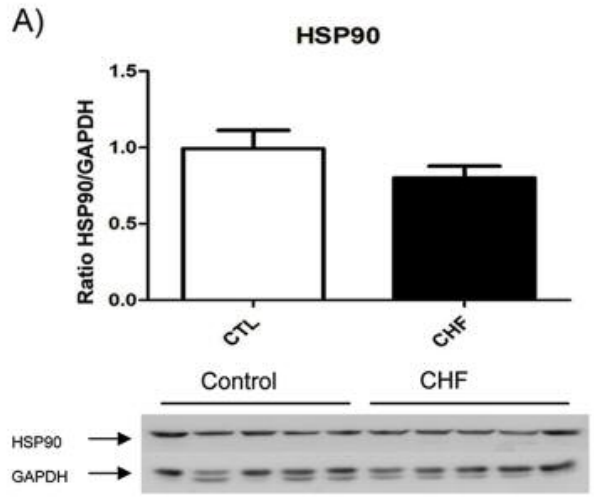

B)
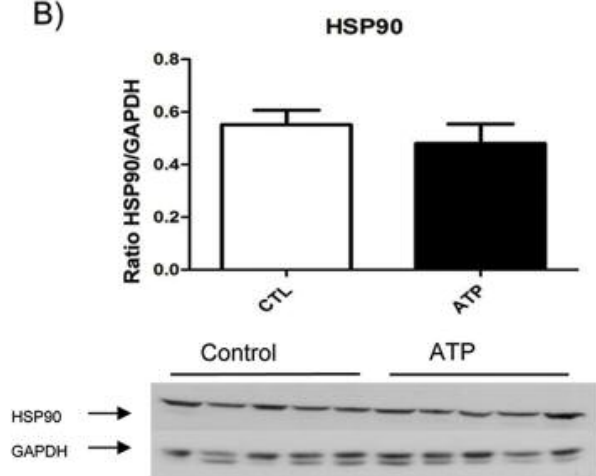

Figure 3: Western Blots and corresponding data showing the relative expression of HSP90 in control dogs $(n=5)$ versus A) Ventricular tachypacing model of CHF $(n=5), p=0.2155$ and B) ATP $(n=5)$, $\mathrm{p}=0.4630$. Protein expression levels were unchanged in either ATP or CHF models.

phosphorylation of at least one serine residue (ser-78) is significantly decreased in the canine model of CHF. Although the phosphorylation of HSP27 also tended to be decreased in the ATP group, these effects were inconsistent between samples and might be attributed to individual variations between the canine subjects. According to the results in our current study, it could be inferred that during sustained periods of AF-promoting conditions, endogenous HSP27 is unlikely to convey a protective effect. Decreased phosphorylation would hinder the cytoprotective role of HSP27 and allow for atrial remodeling, thus promoting $\mathrm{AF}$ maintenance. However, other studies have shown that large oligomers of HSP27 are necessary for it to exhibit chaperone functions, and that phosphorylation causes a dissociation of HSP27 into tetramers, which is not an effective chaperone (12). Therefore, a decrease in phosphorylation, as seen in this present study, could allow for HSP27 to act as a better chaperone in the cytoplasm and provide resistance against stress. The findings that non-phosphorylated HSP27 oligomers confer resistance to the cytotoxic effects of $\mathrm{H}_{2} \mathrm{O}_{2}$, manadione, and TNF $\alpha$, and that phosphorylated mutants of HSP27 convey less of a protective function supports this claim (13).
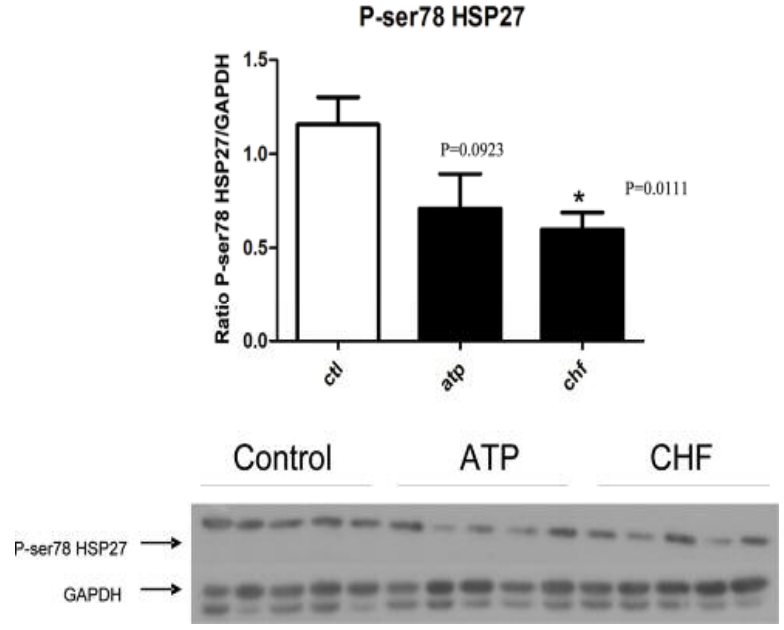

Figure 4: Western Blots and corresponding data showing the relative levels of phosphorylated HSP27 (ser-78) in control dogs $(n=5)$ compared to the ATP group $(\mathrm{n}=5), \mathrm{p}=0.0923$, and the CHF group $(\mathrm{n}=5), \mathrm{p}=0.0111 . * \mathrm{P}<0.05$

It is widely acknowledged that HSP27 and other chaperone proteins are cytoprotective during conditions of stress. However, it is apparent that there is not yet a clear mechanism as to how the phosphorylation status of HSP27 affects its protective function. In our study, we had a statistically significant decrease in phosphorylation in the CHF model of AF. According to our current understanding of HSP27 phosphorylation, as discussed above, these results can be interpreted in two contrasting ways: 1) the observed decrease in phosphorylation, and thus a decrease in HSP27's protective function, allows for atrial remodeling and therefore the maintenance of $\mathrm{AF}$, or 2) the decreased phosphorylation is in fact a protective mechanism and is an attempt to counteract the AF promoting conditions and prevent remodeling.

Our data clearly indicates that phosphorylation of HSP27 has a role in the pathophysiology of AF, however, according to current knowledge, it is impossible to say whether this change is harmful or beneficial. Since this study was not designed to resolve this discrepancy, more research is obviously necessary to determine which of these two contrasting mechanisms is involved in the pathophysiology of AF.

Contrary to data collected from earlier studies using human atrial samples, we failed to detect any change in the level of expression of various HSPs in two canine models of AF. For example, our study found that there was no change in expression of HSP25 (an ortholog of HSP27) while a limited study by Yang et al. detected a trend, which did not reach significance, towards an increase in HSP27 expression in 17 patients with AF, compared to 7 in SR, and concluded that HSP27 may be associated with different stages of AF (7). Similar results were also reported by Brundel et al. who found 
that HSP27 levels were significantly increased in patients with paroxysmal AF compared to patients in SR and with persistent AF (5). The conclusion reached by their study was that increased levels of Hsp27 may protect against myolysis and slow the progression to persistent AF. It should be noted that human studies, especially with a small sample size, do not allow for the same degree of control and reproducibility as animal models. The pathophysiology of AF is complex, and the duration and severity can vary widely between individuals, which would make data unreliable in small sample sizes. Differences between these earlier human studies and our current study could also be the result of other factors including species variations since unlike the former; this study involved the use of two well established canine models. It should also be noted that the canine subjects were not screened for underlying cardiac conditions which may have also contributed to some of the observed variations detected between individual samples. Therefore, the use of a canine model is able to limit, but not entirely eliminate, intersubject variability. Since using animal models allows for a greater degree of control and reproducibility, the results from this study are more reliable compared to those from human studies with a small sample size. To resolve the differences between the human studies and our in vivo canine models, further studies investigating the expression of HSPs during AF are warranted.

\section{CONCLUSION}

Research shows that HSPs have a role in the pathophysiology of atrial fibrillation. Although our data shows that the expression levels of HSPs may remain constant, we demonstrate that there are protein phosphorylation and dephosphorylation events that occur in AF that may have important consequences in its pathophysiology. In the case of HSP27, we detected a significant decrease in its phosphorylation in a canine model of CHF. Also note that other HSPs such as HSP70 and HSP90 are known to be involved in proteinprotein interactions and are modified under various physiological conditions (14). Therefore, this study provides important evidence that requires further investigation into HSP post-translational modifications and protein-protein interactions in $\mathrm{AF}$ and its relevance to the pathophysiology of this condition. It will be necessary to investigate the full scale of HSP modifications during $\mathrm{AF}$ and $\mathrm{AF}$-promoting conditions.

\section{ACKNOWLEDGEMENTS}

We would like to thank the Mach Gaensslen Foundation of Canada for providing the grant for this research project.

\section{REFERENCES}

1. Allessie MA, Boyden PA, Camm AJ, et al. Pathophysiology and Prevention of Atrial Fibrillation. Circulation. 2001;103:769777.

2. Benjamin EJ, Wolf PA, D'Agostino RB, et al. Impact of atrial fibrillation on the risk of death: the Framingham Heart Study. Circulation 1998;98(10):946-52.

3. Wijffels MC, Kirchhof CJ, Dorland R, et al. Atrial Fibrillation begets atrial fibrillation. A study in awake chronically instrumented goats. Circulation 1995;92:1954-1968.

4. Schmitt E, Gehrmann M, Brunet $M$, et al. Intracellular and extracellular functions of heat shock proteins : repercussions in cancer therapy. Journal of Leukocyte Biology 2007;81:15-27.

5. Brundel BJ, Henning RH, Ke L, van Gelder IC, et al. Heat shock protein upregulation protects against pacing-induced myolysis in HL-1 atrial myocytes and in human atrial fibrillation. J Mol Cell Cardiol. 2006 Sep;41(3):555-62.

6. Schafler A, Kirmanoglou K, Balbach J, et al. The expression of heat shock protein 60 in myocardium of patients with chronic atrial fibrillation. Basic Res Cardiol. 2002;97:258-261.

7. Yang M, Tan H, Cheng L, et al. Expression of heat shock proteins in myocardium of patients with atrial fibrillation. Cell Stress and Chaperones. 2007;12(2):142-150.

8. Sakabe M, Shiroshita-Takeshita A, Maguy A, et al. Effects of a heat-shock protein inducer on the atrial fibrillation substrate caused by acute atrial ischemia. Cardiovasc Res. 2008 Jan 31.

9. Cha TJ, Ehrlich JR, Zhang L, Shi YF, Tardif JC, Leung TK, Nattel S. Dissociation between ionic remodeling and ability to sustain atrial fibrillation during recovery from experimental congestive heart failure. Circulation. 2004;109:412- 418.

10. Li D, Fareh S, Leung TK et al. Promotion of atrial fibrillation by heart failure in dogs: atrial remodeling of a different sort. Circulation. 1999;100:87-95.

11. Geum D, Son GH, Kim K. Phosphorylation-dependent cellular localization and thermoprotective role of heat shock protein 25 in hippocampal progenitor cells. Journal of Biological Chemistry. 2002;227:19913-19921.

12. Venkatakrishnan CD, Tewari AK, Moldovan L et al. Heat shock protects cardiac cells from Doxorubicin-induced toxicity by activating p38MAPK and phosphorylation of small heat shock protein 27. Am J Physiol Heart Circ Physiol. 2006;291:H2680-H2691.

13. Rogalla T, Ehrnsperger M, Preville X, et al. Regulation of HSP27 oligomerization, chaperone function, and protective activity against oxidative stress/tumor necrosis factor $\alpha$ by phosphorylation. Journal of Biological Chemistry. 1999;274:18947-18956.

14. Daniel S, Bradley G, Longshaw V, et al. Nuclear translocation of phosphoprotein Hop (Hsp70/hsp90 organizing protein) occurs under heat shock, and its proposed nuclear localization signal is involved in Hsp90 binding. Biochimica et Biophysica Acta. 2008;1783:1002-1014. 